\title{
Manifestações articulares e neoplasias
}

\author{
Articular manifestations and neoplasms \\ Manifestaciones articulares y neoplasias
}

\section{Resumo}

Objetivo: Identificar os estudos relacionados às ocorrências de câncer que tenham manifestações articulares como principal sintomatologia. Metodologia: Trata-se de uma revisão integrativa da literatura, desenvolvida através da análise de artigos relacionados ao tema, em que o levantamento bibliográfico foi realizado através das base de dados Scientific Electronic Library Online, Literatura Latino-Americana e do Caribe em Ciências da Saúde e Google Acadêmico. Utilizou-se, assim, os Descritores Controlados em Ciências da Saúde "manifestações articulares" e neoplasia. Resultados: Após a realização da pesquisa foram identificados 210 artigos e elegeram-se oito publicações depois de aplicados os critérios de inclusão e exclusão e leitura criteriosa dos artigos. Além disso, foram categorizadas evidenciando se havia forte relação da neoplasia com as manifestações articulares até a categorização em que não houve nenhuma relação. Conclusão: Há uma relação entre as neoplasias e as manifestações articulares, bem como, identificou-se que alguns tipos de câncer se manifestam através de doenças articulares visto que, em pacientes com linfoma podem ser consequentes e secundários as manifestações de gota, reação dos tecidos adjacentes ao linfoma ou infiltração sinovial pelo linfoma.

Palavras-chave: Manifestações articulares; Neoplasias; Câncer.

\begin{abstract}
Objective: To identify studies related to occurrences of cancer that have joint manifestations as the main symptom. Methodology: This is an integrative literature review, developed through the analysis of articles related to the topic, in which the bibliographic survey was carried out using the Scientific Electronic Library Online, Latin American and Caribbean Literature in Health Sciences database. and Google Scholar. Thus, the Controlled Descriptors in Health Sciences "articular manifestations" and neoplasia were used. Results: After conducting the research, 210 articles were identified and eight publications were elected after applying the inclusion and exclusion criteria and careful reading of the articles. In addition, they were categorized showing whether there was a strong relationship between the neoplasm and joint manifestations, until the categorization in which there was no relationship. Conclusion: There is a relationship between neoplasms and joint manifestations, as well as, it was identified that some types of cancer manifest through joint diseases since, in patients with lymphoma, gout, tissue reaction can be consequential and secondary. adjacent to lymphoma or synovial infiltration by lymphoma.
\end{abstract}

Keywords: Articular manifestations; Neoplasms; Cancer.

\section{Resumen}

Objetivo: Identificar estudios relacionados con casos de cáncer que tengan como síntoma principal las manifestaciones articulares. Metodología: Se trata de una revisión de literatura integradora, desarrollada a través del análisis de artículos relacionados con el tema, en la cual se realizó el relevamiento bibliográfico utilizando la base de datos Scientific Electronic Library Online, Latin American and Caribbean Literature in Health Sciences y Google Scholar. Así, se utilizaron los Descriptores Controlados en Ciencias de la Salud "manifestaciones articulares" y neoplasias. Resultados: Tras la realización de la investigación, se identificaron 210 artículos y se eligieron ocho publicaciones tras aplicar los criterios de inclusión y exclusión y una lectura atenta de los artículos. Además, se categorizaron mostrando si existía una fuerte relación entre la neoplasia y las manifestaciones articulares, hasta la categorización en la que no hubo relación. Conclusión: Existe una relación entre las neoplasias y las manifestaciones 
articulares, así como, se identificó que algunos tipos de cáncer se manifiestan a través de enfermedades articulares ya que, en pacientes con linfoma, gota, la reacción tisular puede ser consecuencial y secundaria. infiltración por linfoma.

Palabras clave: Manifestaciones articulares; Neoplasias; Cáncer.

\section{Introdução}

Síndromes paraneoplásicas ocorrem quando um câncer causa sintomas incomuns devido a substâncias que circulam na corrente sanguínea. Essas substâncias podem ser hormônios produzidos pelo tumor ou anticorpos produzidos pelo sistema imunológico. Eles podem afetar a função de vários tecidos e órgãos e causar sintomas em locais distantes do tumor. As síndromes paraneoplásicas podem afetar muitos sistemas de órgãos diferentes, incluindo o sistema nervoso e o sistema endócrino (hormônios), causando problemas como mudanças do sistema nervoso, baixo nível de açúcar no sangue, diarreia ou pressão arterial alta (Gale, 2018).

Muitas manifestações reumáticas ocorrem em associação a doenças hematológicas. O que pode justificar é que algumas doenças reumáticas remetam risco de doenças oncohematológicas, como as mielodisplasias, as leucemias, os linfomas e o mieloma múltiplo, que acometer articulações, músculos e vasos, sendo assim connhecidas como doenças para-neoplásicas (Machado, Braz \& Freire, 2014).

Segundo Goulart e Smaletz (2008), as neoplasias que mais se associam às síndromes paraneoplásicas são pulmonar, renal, hapatocelular, leucemias, linfomas, tumores de mama e ovário, gástricos, pancreáticos e reumáticas. Entre as síndromes reumáticas paraneoplásicas mais frequentemente diagnosticadas estão a osteoartropatia hipertrófica, a poliartrite (artrite reumatóide like), a dermatopolimiosite e as vasculites paraneoplásicas. Essas manifestações podem ser concomitantes, preceder ou seguir o diagnóstico do processo tumoral ou sua recorrência; o tratamento da neoplasia frequentemente resulta na regressão das manifestações clínicas (Fam, 2000).

Pode-se observar o frequente acometimento de casos de câncer camuflados por manifestações articulares. Muitas vezes o paciente se queixa de problemas nas articulações e só após uma análise e investigação de especialistas acaba descobrindo se tratar de um câncer (Kontzias, 2020).

Essas manifestações podem surgir a partir de duas situações: tumor primário (benigno ou maligno) ósseo, cartilaginoso, fibroso, do tecido conjuntivo ou de origem mista invadindo diretamente o tecido ósseo, articular ou muscular; tumor ósseo metastático; infiltração maligna da medula óssea; síndromes paraneoplásicas induzidas por tumores distantes via mediadores inflamatórios e eventos adversos às medicações antineoplásicas (Joyce \& Ilaslan, 2018).

$\mathrm{Na}$ maioria dos casos o comprometimento osteoarticular é a primeira manifestação da neoplasia ou prevalecer em relação aos demais sinais e sintomas da doença, sendo muitas vezes diagnosticado como doença reumática, ocasionando demora no diagnóstico e afetando o prognóstico do paciente (Araújo et al., 2018).

Os tumores raramente afetam as articulações a não ser por extensão direta de osso adjacente ou do tumor do tecido mole. No entanto, em duas condições específicas: osteocondromatose e sinovite vilonodular pigmentada, ocorre no revestimento (sinóvia) das articulações. Estes tumores são benignos, mas localmente agressivos. Em geral, ambos os distúrbios afetam uma articulação, com mais frequência no joelho, quadril e podem causar dor e efusão. Os dois são tratados por sinovectomia e remoção de qualquer infraestrutura de corpos estranhos (Joyce \& Ilaslan, 2018).

Além disso, quando pesquisado causas de osteoartropatia hipertrófica secundária em um paciente com dor e tumefação dos joelhos com três meses de evolução, revelou a existência de uma neoplasia pulmonar volumosa, que não apresentou no exame clínico quaisquer manifestações respiratórias (Inês et al., 2002).

Dessa forma, pacientes com dores articulares intensas e diagnósticos de osteroartrose e tendinite, porém com intensidade, ritmo e periodicidade da dor, bem como a falta de resposta aos tratamentos realizados, devem ser investigados para neoplasias. Sendo assim, em casos de dores articulares ou periarticulares intensas, rebeldes a tratamento conservador, 
principalmente em idosos, o diagnóstico de metástase óssea deve ser cogitado, fazendo uma pesquisa com exames laboratoriais, radiografias, cintilografia óssea e, por vezes, tomografia computadorizada e ressonância nuclear magnética (Dancour et al., 2001).

Ainda nesse contexto, um estudo mostrou um caso de neoplasia pancreática, em que artrite e lesões eritematosas nodulares precederam o diagnóstico de doença pancreática. O paciente apresentava artrite em articulações de punhos, joelhos, primeira metatarsofalangiana e tarso bilateralmente, que sentia dor de grande intensidade, eritema e calor havia aproximadamente três semanas, além de febre em torno de $38^{\circ} \mathrm{C}$ de frequência diária e lesões nodulares, eritematosas e dolorosas localizadas em membros inferiores e membros superiores, semelhantes ao eritema nodoso, sem melhora do processo inflamatório ou da dor quando tratada com anti-inflamatórios e corticoides. Deve-se, portanto, estar atento para a possibilidade diagnóstica de doença maligna pancreática, em vigência de paniculite e artrite, mesmo sem queixas abdominais (Sá et al., 2007).

Entre as síndromes reumáticas e manifestações articulares relacionadas ao câncer, tem-se a polimialgia reumática (PMR) que é uma doença reumática inflamatória comum em adultos com 50 anos ou mais, caraterizada por dor e rigidez das cinturas musculares escapular e pélvica, frequentemente associada a elevação dos marcadores inflamatórios e a resposta ao tratamento com corticoides. Nos doentes com manifestações atípicas de PMR ou uma má resposta terapêutica à corticoterapia há indicação para iniciar pesquisa de possível neoplasia oculta. Múltiplas neoplasias parecem estar associadas a PMR, enquanto manifestação paraneoplásica, nomeadamente neoplasias do estômago, rim, pulmão, próstata e do tecido linfohematopoiético (Cabral, 2016).

Outro exemplo de manifestações articulares e neoplasias, temos a dermatomiosite (DM) que é uma doença rara, caracterizada por fraqueza muscular proximal associada a exantema cutâneo típico. A biopsia muscular apresenta lesões inflamatórias compatíveis com miosite, estando associada a um aumento de risco de neoplasia, frequentemente considerada como síndrome paraneoplásica (Castro, Barroso \& Parente, 2013).

Portanto, esta pesquisa busca identificar os estudos relacionados às ocorrências de câncer disfarçadas de manifestações articulares, que muitas vezes fazem com que os médicos façam um diagnóstico divergente com a realidade.

\section{Metodologia}

Trata-se de uma Revisão Integrativa da Literatura (RIL), desenvolvida e baseada nas seis fases do processo de elaboração: $1^{a}$ fase - elaboração da pergunta norteadora; $2^{\mathrm{a}}$ fase - busca ou amostragem da literatura; $3^{\mathrm{a}}$ fase - coleta de dados; $4^{\mathrm{a}}$ fase - análise crítica dos estudos incluídos; $5^{\mathrm{a}}$ fase - discussão dos resultados; $6^{\mathrm{a}}$ fase - apresentação da revisão integrativa (Carvalho, Souza \& Silva, 2010).

Com base na $1^{a}$ fase da construção da pesquisa, partiu-se da seguinte questão norteadora: <<Qual a relação entre as neoplasias e as manifestações articulares?〉>.

O levantamento bibliográfico (2 $2^{\circ}$ etapa), foi realizado através das bases de dados científicas Scientific Electronic Library Online (Scielo), Literatura Latino-Americana e do Caribe em Ciências da Saúde (Lilacs) e Google Acadêmico.

Os critérios de inclusão foram artigos originais sobre o tema pesquisado, no período entre 2015 a 2020, com delineamento experimental (casos clínicos) ou observacional (estudos de caso). Já os critérios de exclusão foram publicações repetidas e publicações que não estavam no idioma português.

Utilizou-se a terminologia em saúde consultada nos Descritores em Ciência da Saúde (DeCS) "manifestações articulares" e neoplasia. Com a pesquisa bibliográfica ocorrida em outubro de 2020 obteve-se uma amostra inicial de 210 publicações. Os critérios de exclusão possibilitou a determinação da amostragem final, constituída por 45 artigos. Foi realizada 
uma leitura criteriosa, com a finalidade de refinar as amostras e selecionar os artigos mais pertinentes ao tema e que responderia a questão norteadora, finalizando com 8 artigos (Figura 1).

É oportuno ressaltar ainda que o intervalo temporal determinado para seleção foi entre os anos de 2015 a 2020. Optouse por este período por tratar-se de um assunto cada vez mais atual e sua utilização vem se intensificando nos últimos anos.

Devido à pesquisa ser qualitativa, a análise dos dados foi feita através da constatação, no material pesquisado sobre o tema da pesquisa. As informações foram divididas de acordo com os objetivos dessa pesquisa e distribuídas como respostas de acordo com a sequência de assuntos.

Em seguida, já com a amostra final determinada, realizou-se a leitura analítica, cuja finalidade foi ordenar e sumarizar as informações contidas nos artigos selecionados para responder aos objetivos da pesquisa possibilitando, posteriormente, a organização dos dados coletados. As variáveis contempladas para a caracterização geral foram: Autores/ano, título do artigo, base de dados (BD), título do periódico, idioma, país. Foi usado também caracterização metodológica com outras variáveis como tipo de pesquisa, população-alvo, tamanho amostral.

Dando seguimento a RIL, executou-se a interpretação e avaliação dos resultados, o que propiciou compilar das informações de cada estudo e a síntese da revisão. A presente revisão de literatura assegura os aspectos éticos, garantindo a autoria dos artigos pesquisados, utilizando para citações e referências dos autores as normas da American Psychological Association (APA). 
Figura 1 - Fluxograma da revisão descritiva. Patos, 2021.
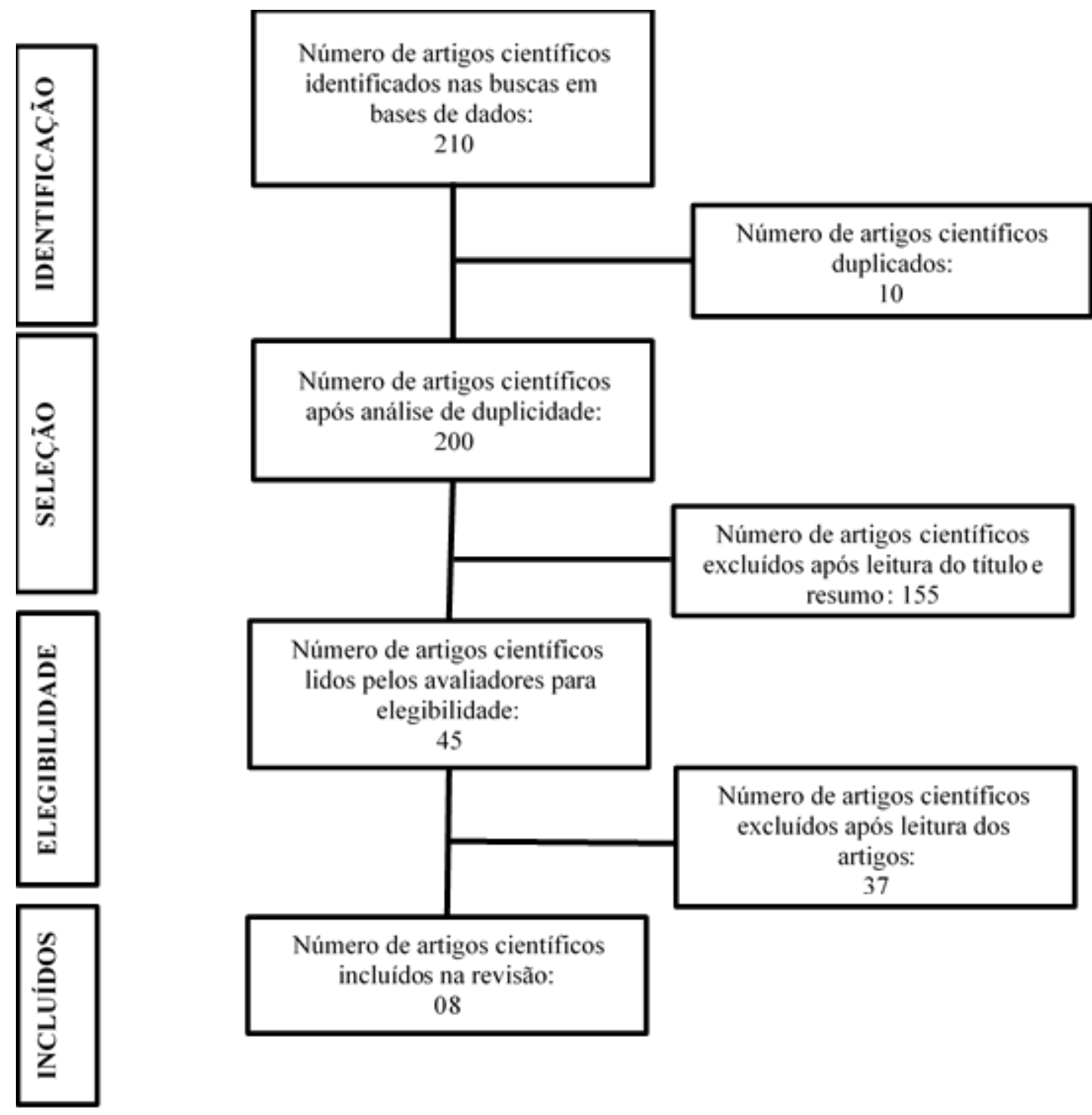

Fonte: Autores.

\section{Resultados}

A categorização geral dos artigos foi feita com base nas variáveis autores/ano, título do artigo, base de dados, título do periódico, idioma e país, como mostra o Quadro 1.

Em relação ao idioma da publicação do artigo, observou-se que $100 \%$ (n=8) estavam em português, bem como o país de origem dos artigos selecionados nos artigos. Quanto a base de dados, a maioria era da Literatura Latino-Americana e do Caribe em Ciências da Saúde (Lilacs), tendo uma porcentagem de 50\% (n=4), em sequência vem a base de dados Scientific Electronic Library Online (Scielo) com 37,5\% ( $\mathrm{n}=3$ ) das publicações selecionadas, por fim aparece o Google Acadêmico com 12,5\% (n=1). Em relação ao Título do Periódico, os periódicos Arquivos Brasileiros de Cardiologia e Revista Brasileira de Reumatologia foram os que tiveram maior porcentagem, ambos com $25 \%(\mathrm{n}=2)$. 
Research, Society and Development, v. 10, n. 9, e52810918474, 2021

(CC BY 4.0) | ISSN 2525-3409 | DOI: http://dx.doi.org/10.33448/rsd-v10i9.18474

Quadro 1: Caracterização geral dos artigos selecionados para compor a RIL. Patos, 2021.

\begin{tabular}{|c|c|c|c|c|c|}
\hline Autores/Ano & Título do Artigo & BD & Título do Periódico & Idioma & País \\
\hline Oliveira (2007) & Alterações Osteoarticulares & $\begin{array}{l}\text { Google } \\
\text { Acadêmico }\end{array}$ & Ciências da Saúde & Português & Brasil \\
\hline $\begin{array}{l}\text { Liphaus, Terrari \& } \\
\text { Sacchetti (2008) }\end{array}$ & $\begin{array}{l}\text { Manifestações músculo- } \\
\text { esqueléticas nas doenças não } \\
\text { reumatológicas. }\end{array}$ & Lilacs & $\begin{array}{l}\text { Revista de Saúde } \\
\text { Coletiva }\end{array}$ & Português & Brasil \\
\hline Hamerschlak (2008) & $\begin{array}{l}\text { Manifestações reumáticas } \\
\text { associadas a doenças } \\
\text { oncohematológicas. }\end{array}$ & Lilacs & $\begin{array}{l}\text { Sociedade de Pediatria } \\
\text { de São Paulo }\end{array}$ & Português & Brasil \\
\hline Campos et al. (2008) & $\begin{array}{l}\text { Comprometimento } \\
\text { musculoesquelético como } \\
\text { primeira manifestação de } \\
\text { neoplasias }\end{array}$ & Scielo & Rev. Assoc. Med. Bras. & Português & Brasil \\
\hline $\begin{array}{l}\text { Machado, Braz \& } \\
\text { Freire (2014) }\end{array}$ & 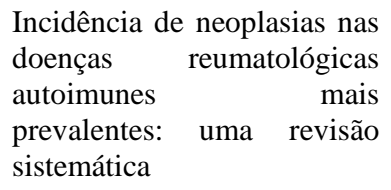 & Lilacs & Rev. Bras. Reumatol. & Português & Brasil \\
\hline Fonseca et al. (2017) & $\begin{array}{l}\text { Sinais e sintomas sugestivos } \\
\text { de doenças reumáticas como } \\
\text { primeira manifestação de } \\
\text { doenças neoplásicas na } \\
\text { infância: Implicações no } \\
\text { diagnóstico e prognóstico. }\end{array}$ & Lilacs & Rev. Bras. Reumatol. & Português & Brasil \\
\hline Ferrari (2020) & $\begin{array}{lll}\text { COVID-19: } & & \text { Dados } \\
\text { Atualizados e sua } & \text { Relação } \\
\text { Com o } & \text { Sistema } \\
\text { Cardiovascular } & & \end{array}$ & Scielo & Arq Bras Cardiol. & Português & Brasil \\
\hline Costa et al. (2020) & $\begin{array}{l}\text { O coração e a Covid-19: O } \\
\text { que o cardiologista precisa } \\
\text { saber. }\end{array}$ & Scielo & Arq Bras Cardiol. & Português & Brasil \\
\hline
\end{tabular}

Fonte: Autores.

Ainda em relação aos artigos selecionados foi possível identificar a metodologia, caracterizando-os de acordo com o tipo de pesquisa, público-alvo dos estudos selecionados e o tamanho amostral (Quadro 2).

Dessa forma, de acordo com o tipo de pesquisa, era pesquisa bibliográfica 37,5\% (n=3) dos artigos, seguido por análise retrospectiva e também por pesquisa exploratória, com ambas possuindo porcentagem de $25 \%$ ( $\mathrm{n}=2)$. A população-alvo de cada artigo era diversificada, sendo 25\% ( $\mathrm{n}=2$ ) sobre populações que tiveram COVID-19. Em relação ao tamanho amostral, $62,5 \%$ ( $\mathrm{n}=5$ ) não possuía especificações. 
Quadro 2: Caracterização metodológica dos artigos selecionados para compor a RIL. Patos, 2021.

\begin{tabular}{|l|l|l|l|}
\hline Autores/Ano & Tipo de pesquisa & População-alvo & Tamanho amostral \\
\hline Oliveira (2007) & Analítica & $\begin{array}{l}\text { Indivíduos com alterações } \\
\text { osteoarticulares }\end{array}$ & Sem especificações \\
\hline $\begin{array}{l}\text { Liphaus, Terrari \& } \\
\text { Sacchetti (2008) }\end{array}$ & Bibliográfica & População em geral Sem especificações \\
\hline Hamerschlak (2008) & Bibliográfica & $\begin{array}{l}\text { População com manifestações } \\
\text { reumáticas associadas a doenças } \\
\text { onchematológicas }\end{array}$ & Sem especificações \\
\hline Campos et al. (2008) & Análise retrospectiva & $\begin{array}{l}\text { Unidade de Reumatologia do } \\
\text { Instituto da Criança - FMUSP }\end{array}$ & 4876 pacientes \\
\hline $\begin{array}{l}\text { Machado, Braz \& Freire } \\
\text { (2014) }\end{array}$ & Bibliográfica & $\begin{array}{l}\text { Artigos científicos sobre incidência } \\
\text { de neoplasia e doenças } \\
\text { reumatológicas autoimunes }\end{array}$ & artigos \\
\hline Fonseca et al. (2017) & Análise retrospectiva & $\begin{array}{l}\text { Pacientes atenios na Clínica } \\
\text { terciária de Reumatologia Pediátrica } \\
\text { do Hospital da Faculdade de } \\
\text { Medicina de Ribeirão Preto com } \\
\text { diagnóstico de câncer }\end{array}$ & \\
\hline Ferrari (2020) & Exploratória & $\begin{array}{l}\text { Diagnosticados com SARSCov2 } \\
\text { Costa et al. (2020) }\end{array}$ & $\begin{array}{l}\text { População contaminada com } \\
\text { COVID-19 que apresenta alterações } \\
\text { cardiovasculares Sespecificações }\end{array}$ \\
\hline
\end{tabular}

Fonte: Autores.

Ainda, a partir desta Revisão Integrativa da Literatura também foi possível realizar a categorização dos achados obtidos nas publicações selecionadas, evidenciando se havia forte relação da neoplasia com as manifestações articulares até a categorização em que não houve nenhuma relação.

Assim, na categoria 1 estariam os artigos que apresentaram muito forte relação da neoplasia com as manifestações articulares, na categoria 2 estariam os artigos com forte relação da neoplasia com as manifestações articulares, na categoria 3 os de média relação da neoplasia com as manifestações articulares, na categoria 4 estariam os de baixa relação da neoplasia com as manifestações articulares e, por fim, na categoria 5 os artigos em que não há nenhuma relação da neoplasia com as manifestações articulares.

De acordo com os artigos selecionados, dispostos no Quadro 3, constatou-se que a maioria das publicações que compuseram esta revisão se encaixou na categoria 2 de forte relação da neoplasia com as manifestações articulares, com um percentual de $50 \%(n=4)$. As demais categorias obtiveram, portanto, $12,5 \%(n=1)$.

De acordo com o Quadro 3, foi possível também ver a classificação do artigo selecionada na categoria proposta. Após isso, fez-se a classificação dos artigos contidos na publicação, seu número e portagem desses artigos. 
Quadro 3: Classificação através da categorização dos achados da RIL. Patos, 2021.

\begin{tabular}{|l|l|l|l|}
\hline \multicolumn{1}{|c|}{ Categoria dos estudos } & Autores/Ano & N & $\%$ \\
\hline $\begin{array}{l}4-\text { baixa relação da neoplasia com as } \\
\text { manifestações articulares }\end{array}$ & Oliveira (2007) & $2 \%$ \\
\hline $\begin{array}{l}1-\text { muito forte relação da neoplasia com as } \\
\text { manifestações articulares }\end{array}$ & $\begin{array}{l}\text { Liphaus, Terrari \& } \\
\text { Sacchetti (2008) }\end{array}$ & 30 & $20,5 \%$ \\
\hline $\begin{array}{l}2-\text { forte relação da neoplasia com as } \\
\text { manifestações articulares }\end{array}$ & Hamerschlak (2008) & 20 & $12 \%$ \\
\hline $\begin{array}{l}3-\text { média relação da neoplasia com as } \\
\text { manifestações articulares }\end{array}$ & Campos et al. (2008) & 10 & $2 \%$ \\
\hline $\begin{array}{l}5-\text { não há nenhuma relação da neoplasia com as } \\
\text { manifestações articulares }\end{array}$ & $\begin{array}{l}\text { Machado, Braz \& Freire } \\
\text { (2014) }\end{array}$ & 1 & $20 \%$ \\
\hline $\begin{array}{l}2-\text { forte relação da neoplasia com as } \\
\text { manifestações articulares }\end{array}$ & Fonseca et al. (2017) & 28 & $20 \%$ \\
\hline $\begin{array}{l}2-\text { forte relação da neoplasia com as } \\
\text { manifestações articulares }\end{array}$ & Ferrari (2020) & 28 & $18.5 \%$ \\
\hline $\begin{array}{l}2-\text { forte relação da neoplasia com as } \\
\text { manifestações articulares }\end{array}$ & Costa et al. (2020) & 59 & 28 \\
\hline
\end{tabular}

Fonte: Autores.

\section{Discussão}

Frente ao exposto, buscando identificar a relação entre as neoplasias e as manifestações articulares, foi possível analisar a categoria de 1 a 5 , onde 1 forte relação da neoplasia com as manifestações articulares, de modo que consequentemente 5 não há nenhuma relação, considerando os estudos de Fonseca et. al. (2017), Liphaus, Terrari e Sacchetti (2008), Hamerschlak (2008), Ferrari (2020), Costa et. al. (2020), Oliveira (2007), Campos et. al. (2008), e ainda, Machado, Braz e Freire (2014).

$\mathrm{Na}$ assertiva de Fonseca et. al. (2017), aduz que em seus estudos dentre 250 pacientes analisad0os durante todo o período dos seus estudos, cerca de $2 \%$ apresentaram posteriormente diagnóstico de câncer, de modo que $60 \%$ desses, apresentaram em algum momento sintomas como artrite.

Na visão de Liphaus, Terrari e Sacchetti (2008), frente a premência de entendimento se há uma relação entre as neoplasias e as manifestações articulares, é sabido alegar que algumas doenças podem apresentar de maneira evidente diversas manifestações no que concerne o musculoesqueléticas, seja em curto, médio ou longo prazo.

Vale ressaltar ainda, que dentre essas manifestações pode-se mensurar dor em membros, bem como, fraqueza muscular, e ainda lombalgia são as mais evidentes apontada pelos estudos de Liphaus, Terrari e Sacchetti (2008), corroborando assim com o entendimento de que diversas doenças, inclusive as doenças onchematológicas, podem apresentam variados sintomas que afetam o musculoesquelético.

Já para Hamerschlak (2008), o mesmo ressalta nos desfechos de seus estudos que alguns mieloma, podem apresentar várias manifestações ósseas demasiadamente dolorosas para os indivíduos, de modo que acaba implicando inclusive em diversas fraturas ósseas provocadas pela doença. Tal assertiva remete ao entendimento, de que em conformidade com os desfechos de Hamerschlak (2008), as manifestações reumáticas estão associadas a um risco de doenças oncohematológicas.

Ainda sob a ótica de Liphaus, Terrari e Sacchetti (2008) é importante mensurar que dentre as doenças sistêmicas que podem vir a apresentar sintomas ou manifestações musculoesqueléticas, pode-se ressaltar as doenças neoplásicas como tumores ósseos, leucemia, linfoma, e ainda, neuroblastoma. 
Em contrapartida, de acordo com os achados de Ferrari (2020), Costa et. al. (2020), seus estudos corroboram com a temática reforçando que em casos de Covid-19 e doenças como o câncer, é possível apresentar um maior risco de manifestações articulares, comprometendo inclusive a longo tempo as articulações dos pacientes acometidos pelas doenças de câncer e Covid-19, pois podem comprometer as defesas do hospedeiro, remetendo a sequelas.

É importante inferir que de acordo com a perspectiva de Hamerschlak (2008), e Fonseca et. al. (2017), considera-se que no que concerne as doenças consideradas como oncohematológicas podem vir a apresentar algum tipo de manifestação reumática em relação as articulações dos indivíduos portadores da doença, e ainda, no que tange os músculos e vasos.

Observou-se ainda, sob a ótica de Hamerschlak (2008), e Fonseca et. al. (2017), que dentre 250 pacientes analisados, dos que apresentaram doença oncológica como neoplasia, e conforme já mensurado, $60 \%$ dessa população de pacientes estuada apresentou artrite, além de ser significativo o número de pacientes que necessitaram de biópsia óssea para o diagnóstico final.

Na perspectiva de Oliveira (2007), é sabido alegar que dentre as doenças que acometem em manifestações articulares de maneira significativa podendo considerar a associação, podem estar atrelados a diversos quadros clínicos associados como manifestações cardiovasculares, e diante disso, é de fundamental importância considerar todos os fatores e características.

Vale inferir, que Campos et. al. (2008), em seus estudos, evidencia que diversos pacientes apresentem as queixas musculoesqueléticas, podendo assim associar essas queixas as manifestações iniciais, que por sinal, são bastante frequentes em relação a neoplasia, de modo que esse tipo de manifestação precisa ser levado em consideração no que concerne o diagnóstico das doenças reumatológicas.

Em contrapartida, é correto afirmar que os estudos de Machado, Braz e Freire (2014), ressaltam que há pouca evidência de pacientes com artrite e manifestações nas articulações em relação ao risco de câncer, de modo que não há como afirmar de maneira precisa essa associação, mas que há um aumento considerável de riscos de desenvolvimento de neoplasia pulmonar. Contudo, considera-se que pode haver associação, mas que não aumenta o risco da doença.

Por fim, vale aludir, que dentre os procedimentos devidamente utilizados pela equipe médica para que se possa identificar doenças articulares, Hamerschlak (2008), ressalta que os testes radiológicos podem ser utilizados com a finalidade de mensurar o grau de destruição óssea das doenças oncohematológicas, assim como a ressonância magnética.

Logo, entende-se sob a perspesctiva de Campos et. al. (2008), os seus estudos corroboram salientando que por meio dos métodos de imagem é possível identificar as lesões líticas nos ossos através de radiografia de ossos, o que por certo, pode viabilizar uma visão mais ampla sobre os sintomas musculoesqueléticos relacionados as doenças caracterizadas como onchematológicas.

Por fim, Hamerschlak (2008), Campos et. al. (2008), Fonseca et. al. (2017), compreendem em seus estudos abrangendo as suas populações e analises, que há uma associação considerável entre as neoplasias e as manifestações pertinentes as articulações, de modo que é possível que alguns tipos de câncer possam vir a se manifestar por meio de doenças articulares.

\section{Considerações Finais}

De acordo com a pesquisa, pudemos identificar uma relação entre as neoplasias e as manifestações articulares. Foram analisados estudos sobre manifestações articulares relacionadas ao câncer. Identificou-se alguns tipos de câncer que se manifestam inicialmente através de doenças articulares. Sintomas articulares em pacientes com linfoma podem ser consequentes e secundários a manifestações de gota, reação dos tecidos adjacentes ao linfoma ou infiltração sinovial pelo linfoma. 
Uma variedade de fenômenos de patogêneses autoimunes e reumatológicas pode ocorrer nas síndromes mielodisplásicas, como artrite monoarticular, policondrites, fenômeno de Raynaud, síndrome de Sjogren e vasculites.

As síndromes paraneoplásicas e o câncer de pulmão constituem grandes desafios no que tange ao tratamento, sobretudo, em sala de emergência. A fase inicial no atendimento é condição essencial ao bom prognóstico e melhora da qualidade de vida relacionada a saúde de determinados pacientes. Portanto, a realidade do bom atendimento e também do diagnóstico correto se liga a profissionais capacitados.

Pode-se, portanto, pontuar que o conhecimento médico sobre as manifestações articulares atrelado ao surgimento de neoplasias, é imprescindível para a manutenção da saúde dos pacientes que possuem doenças reumatológicas ou apenas sintomatologias de manifestações articulares, uma vez que alguns tipos de câncer que se manifestam inicialmente através de doenças articulares

Faz-se necessário que mais estudos sejam estimulados no meio científico em relação a manifestações articulares e neoplasias. Não obstante, devem-se ser feitos estudos que mostrem o quanto a prática da não relação entre esses temas, pode afetar o prognóstico e a qualidade de vida dos pacientes.

\section{Referências}

Araújo, P. I. C. et al. (2007). Doença Falsiforme com comprometimento. Revista Brasileira de Hematologia e Hemoterapia, 29 (3).

Cabral, J. C. (2016). Polimialgia reumática como síndrome paraneoplásica. FMUC Medicina - Tese de Mestrado. http://hdl.handle.net/10316/34622.

Campos, L. M. (2008) Comprometimento musculoesquelético como primeira manifestação de neoplasias. Revista da Associação Médica Brasileira, 54 (2).

Carvalho, R., Souza, M. T. S. \& Silva, M. T. (2010). Revisão integrativa: o que é e como fazer. Einstein, 8(1 Pt 1):102-106.

Castro, A., Barroso, A., \& Parente, B. (2013). Dermatomyositis as the first manifestation of a lung tumor. Revista Portuguesa de Pneumologia (English Edition), 19 (4), 179-183.

Costa, I. B. S. da S. et. al. (2020). O coração e a Covid-19: O que o cardilogista precisa saber. Arquivos Brasileiros de Cardiologia, 114 (5), 805-816.

Dancour, M. A. A. et al. (2001). Manifestações articulares de neoplasias sólidas: a propósito de dois casos e revisão da literatura. Revista Brasileira De Reumatologia, 41(3), 195-199.

Fam A.G. (2000). Paraneoplastic rheumatic syndromes. Baillieres Best Practice \& Research, Clinical Rheumatology, 14 (3),515-33.

Ferrari, F. (2020). Covid19: Dados atualizados e sua relação com o sistema cardiovascular. Ponto de vista. Arquivos Brasileiros de Cardiologia, 114 (5), 823826.

Fonseca, M. B. et al. (2017). Sinais e sintomas sugestivos de doenças reumáticas como primeira manifestação de doenças neoplásicas na infância: implicações no diagnóstico e prognóstico. Revista Brasileira de Reumatologia, 57 (4), 330-337.

Gale, R. P. (2018). Síndromes paraneoplásicas - Hematologia e oncologia. Manuais MSD edição para profissionais. https://www.msdmanuals.com/ptbr/profissional/hematologia-e-oncologia/visão-geral-sobre-câncer/síndromes-paraneoplásicas

Goulart, L.S.S.A. \& Smaletz, O. (2008). Síndromes reumáticas paraneoplásicas. Einstein, 6 (Supl 1):S98-S101.

Hamerschlak, N. (2008). Manifestações reumáticas associadas a doenças oncohematológicas. Einstein, 6 (Supl 1):S89-S97.

Inês, L. et al. (2002). Artropatia dos joelhos como manifestação inicial de neoplasia pulmonar. Acta Reumatologia Portuguesa, 27(2):137-141.

Joyce, M. J. \& Ilaslan, H. (2018). Tumores ósseos malignos primários - Distúrbios dos tecidos conjuntivo e musculoesquelético. Manuais MSD edição para profissionais. https://www.msdmanuals.com/pt-br/profissional/distúrbios-dos-tecidos-conjuntivo-e-musculoesquelético/tumores-dos-ossos-e-das articulações/tumores-ósseos-malignos-primários

Kontzias, A. (2020). Osteoartrite (OA) - Distúrbios ósseos, articulares e musculares. Manual MSD - Versão Saúde para a família. https://www.msdmanuals.com/pt-br/casa/dist\%C3\%BArbios-\%C3\%B3sseos,-articulares-e-musculares/dist\%C3\%BArbios-articulares/osteoartrite-oa

Liphaus, B. L., Terreri, M. T. R. A, Sacchetti, S. B. (2008). Manifestações músculo-esqueléticas nas doenças não reumatológicas. Sociedade de Pediatria de São Paulo. https://www.spsp.org.br/2008/04/22/manifestacoes_musculoesqueleticas_nas_doencas_nao_reumatologicas/

Machado, R. I. L., Braz, A. de S. \& Freire, E. A. M. (2014). Incidência de neoplasias nas doenças reumatológicas autoimunes mais prevalentes: uma revisão sistemática. Revista Brasileira de Reumatologia, 54 (2): 131-139.

Oliveira, S. M. de. (2007). Alterações osteoarticulares. IV Simpósio Internacional de Hemoglobinopatias. http://www.hemorio.rj.gov.br/Html/ pdf/palestras_2007/Alteracoes_osteoarticulares.pdf

Sá, R. G. d., Teixeira, C. M. L. d. O., Machado, L. G., Bonfante, H. d. L., Chaves, A. M. C., Chebli, J. M. F., \& Oliveira, R. B. d. (2007). Poliartrite e nódulos subcutâneos como primeira manifestação de carcinoma de pâncreas. Revista Brasileira de Reumatologia, 47(5), 387-389. 